\title{
A search for the locus of information overload in pigeon compound matching-to-sample performance
}

\author{
MICHAEL F. BROWN \\ Villanova University, Villanova, Pennsylvania
}

\begin{abstract}
Five pigeons matched element and compound samples in a symbolic matching-to-sample procedure. In Experiment 1, the sample duration varied within each session, ranging from .125 to 8 sec. Element matching accuracy was superior to compound matching accuracy, and matching accuracy improved with sample duration; but there was no evidence for the convergence of element and compound matching accuracy with long sample durations. In Experiment 2, the sample-to-test delay varied from 0 to $4 \mathrm{sec}$. The superiority of element matching accuracy over compound matching accuracy did not vary as a function of delay length. These replications of previous failures to find interactions between sample type and temporal parameters in determining matching accuracy place important constraints on explanations of element superiority in pigeon matching-to-sample performance.
\end{abstract}

The present experiments were carried out in an attempt to solve a dilemma in the literature on the element superiority effect in pigeon matching-to-sample (MTS). It has been consistently reported that element matching accuracy is superior to compound matching accuracy (see, e.g., Maki \& Leith, 1973; Roberts \& Grant, 1978; Santi, Grossi, \& Gibson, 1982). This has been argued to result from information overload-that is, from the additional information that is provided by a compound sample (see, e.g., Riley \& Leith, 1976). Recent evidence favors this information overload hypothesis over several competing explanations of the element superiority effect (Brown \& Morrison, 1990). The favored version of the information overload view points to information uptake as the locus of information overload (Riley \& Leith, 1976). That is, information overload occurs as a result of shared attention at the time of sample presentation. Attention must be distributed among the two dimensions of a compound sample, whereas it can be allocated entirely to the single dimension of an element sample. A second possible locus of information overload is memory storage (Grant, 1981). Memory load might be higher following a compound sample than following an element sample.

However, several experiments, following similar logic, have failed to provide evidence for either of these versions of the information overload hypothesis. If information overload occurs at the time of sample presentation (information uptake), then the element superiority effect should be attenuated with longer sample durations. There have been a number of reported failures to find this

This work was supported by National Institute of Mental Health Grant MH42646. The author thanks Spencer Morrison for his assistance in conducting the experiments. Correspondence concerning this article should be sent to Michael F. Brown, Department of Psychology, Villanova University, Villanova, PA 19085. predicted effect (Brown \& Morrison, 1990; Grant \& MacDonald, 1986; Lamb \& Riley, 1981; Riley, 1984; Santi et al., 1982). Likewise, if information overload occurs during the storage of information between sample presentation and test response, then the element superiority effect should increase when a delay is inserted between the sample and presentation of the test stimuli. This effect has not been found (Cox \& D'Amato, 1982; Roberts \& Grant, 1978). Thus, while on the one hand information overload is currently the favored explanation of the element superiority effect, on the other hand, attempts to specify the locus of that overload have been unsuccessful.

A possible resolution of this dilemma involves three limitations of previous experiments. First, the experiments done to date in which sample duration and/or delay length have been manipulated in the context of element/compound MTS have involved the "true" matching procedure, in which the test stimuli are physically identical to the element sample stimuli. (One exception is an experiment reported by Brown \& Morrison, 1990, in which sample duration was manipulated.) The use of true matching introduces an artifact that can lower compound matching levels ("generalization decrement"; see Cox \& D'Amato, 1982; Maki, Riley, \& Leith, 1976; and Roberts \& Grant, 1978). The maintenance of the element superiority effect at long sample durations and short delays might depend on this artifact. Second, previous experiments have involved subjects trained with element samples prior to the introduction of compound samples. This training history has been shown to be involved in the element superiority effect (Brown \& Morrison, 1990; Grant \& MacDonald, 1986). Thus, this factor might constrain the extent to which element and compound matching accuracy converge at long sample durations (and short delays). Finally, unpublished observations made by Brown and Cook indicate that, with long sample durations, pigeons cease pecking the sample 
prior to the end of the sample presentation period. Thus, long sample durations might not support the additional sample processing that is necessary to eliminate information overload during information uptake. This possibility is supported by the observation of decreases in matching accuracy at very long sample durations (Grant \& MacDonald, 1986; Riley, 1984; Santi et al., 1982).

The present experiments were designed to overcome these three limitations, thereby allowing information overload during information uptake or during information storage to be revealed. First, the experiments involved "symbolic" MTS to eliminate the possibility of generalization decrement (Brown \& Morrison, 1990). Second, although the present subjects had been trained with element samples, their performance had previously been compared to that of a group trained with compound samples (Brown $\&$ Morrison, 1990). This greatly reduces the possibility that element superiority in the present subjects can be attributed to a training artifact. Finally, in the present experiments, sample duration and delay length were manipulated within sessions, rather than between sessions. In Experiment 1 , the sample was terminated by the first peck following the scheduled (but unpredictable) sample duration. It was expected that this technique would result in high, steady rates of sample responding throughout even long sample presentation periods. Pecks to the sampe were measured in order to determine whether or not this occurred.

\section{EXPERIMENT 1}

In the first experiment, sample duration was manipulated within each session in a symbolic MTS procedure. A series of three experimental phases involved the use of different sample durations, ranging from .125 to $8 \mathrm{sec}$. Within each phase, four different sample durations were presented. In addition to choice accuracy with element and compound samples, peck rate to the sample was measured so that any differences in peck rate over the course of the sample presentation period, as well as among trial types, could be detected.

\section{Method}

Subjects. The subjects were 5 White Carneaux pigeons (Columba livia). They were maintained in individual cages, with free access to grit and water in a vivarium with a 12:12-h light:dark (LD) cycle. They were maintained at $80 \%-85 \%$ of free-feeding weights. Immediately prior to the present experiment, the subjects had completed the experiments described by Brown and Morrison (1990). They had been in the "element-trained" group in those experiments. Sessions occurred during the light phase of the LD cycle.

Apparatus. The experiment was carried out in three identical threekey pigeon chambers. These chambers are described in Brown and Morrison (1990).

Procedure. Because the subjects had previously participated in similar experiments, no training was necessary. Each pigeon had previously been assigned a set of sample element-to-test stimulus mappings, such that for each sample element, one of four form stimuli was designated as correct. These assignments can be found in Brown and Morrison (1990, Table 1).

Sessions consisted of 128 matching-to-sample trials. On each trial, the center key was first illuminated with white light. A single peck to this warning signal produced presentation of the sample stimulus on the center key. The sample remained for the scheduled sample duration (see below). All pecks to the sample were recorded in terms of the time from the beginning of the sample period to the peck. The sample was replaced by presentation of two test stimuli on the side keys. The two presented test stimuli were always mapped onto sample elements from the same dimension. That is, the test was either a choice between the two test stimuli mapped onto the two color values or a choice between the two test stimuli mapped onto the two-line orientation values. A single peck to the correct test stimulus resulted in 2.5-sec activation of the food hopper. A peck to the incorrect test stimulus terminated the trial. Trials were separated by a 20 -sec intertrial interval.

Sixty-four trials in each session involved element samples, and the remaining 64 trials involved compound samples. For element trials, the identity of the sample and the location of the correct test stimulus (left vs. right) were counterbalanced within each level of sample duration. For compound trials, the identity of the sample, the dimension (color vs. line) represented by the test, and the location of the correct test stimulus were counterbalanced within each level of sample duration.

During each of the first three phases of the experiment, four different sample durations were presented in a counterbalanced fashion within each session. In the first phase of the experiment, sample durations of $.5,1,2$, and $4 \mathrm{sec}$ were used. This phase lasted 20 sessions. In the second phase (10 sessions), sample durations of $.125, .25, .5$, and $1 \mathrm{sec}$ were used. In the third phase (20 sessions), sample durations were .125 , $.5,2$, and $8 \mathrm{sec}$.

\section{Results}

Figure 1 shows mean matching accuracy for element and compound trials as a function of sample duration during each of the first three phases of the experiment. An analysis of variance (ANOVA: sample duration $\times$ sample type) was carried out for each experimental phase, using the mean choice accuracy of each subject at each level of sample type and sample duration. The data from Phase 1 included an effect of sample duration $[F(3,12)=15.9$, $p<.001]$ and an effect of sample type $[F(1,4)=13.8$, $p<.05$ ), but no interaction between these variables $[F(3,12)<1]$. During Phase 2, there was an effect of sample duration $[F(3,12)=19.0, p<.001]$, as well as an effect of sample type $[F(1,4)=18.0, p<.05]$. There was also a marginally significant interaction between sample type and sample duration during Phase $2[F(3,12)=$ $3.14, p=.06$ ]. The form of this interaction is clearly not the predicted convergence of element and compound matching accuracy with long sample durations. During Phase 3, there was an effect of sample duration $[F(3,12)=$ $21.1, p<.001]$ and an effect of sample type $[F(1,4)=$ $20.2, p<.01]$, but no interaction $[F(3,12)<1)$.

Sample peck rates during the third experimental phase were analyzed by dividing the sample presentation period into bins of $.5 \mathrm{sec}$ each and determining the sample peck rate of each subject in each bin at each level of sample duration and sample type. Mean peck rates are shown in Figure 2 for the three longest sample durations. No sample pecks occurred when the sample was presented for $.125 \mathrm{sec}$. Presumably, there is insufficient time to execute a peck within .125 sec following the peck to the warning signal. Separate ANOVAs (bin $\times$ sample type) were conducted on the data from the three longest sample durations. When the sample duration was $.5 \mathrm{sec}$, there was no difference in peck rate to element and compound samples $[F(1,4)<1]$. When the sample duration was $2 \mathrm{sec}$, there was a significant effect of bin $[F(3,12)=4.4$, 

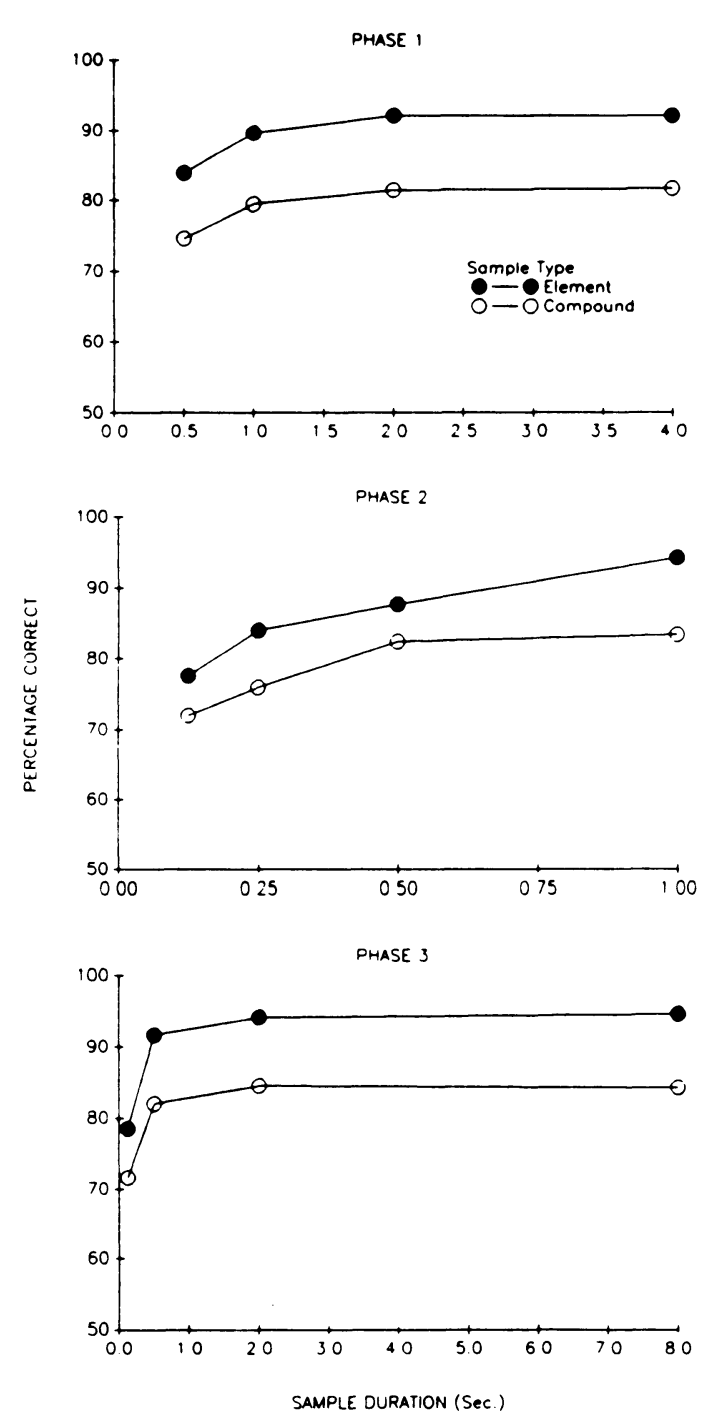

Figure 1. Mean matching accuracy as a function of sample duration for element and compound trials during Phases 1 (top panel), 2 (middle panel), and 3 (bottom panel) of Experiment 1 .

$p<.05]$. Although there was no main effect of sample type $[F(1,4)=1.5]$, there was an interaction between sample type and bin $[F(3,12)=5.8, p=.01]$. This interaction apparently resulted from a difference in peck rates to element and compound samples that was limited to late in the sample presentation period. When the sample duration was $8 \mathrm{sec}$, there was a significant effect of bin $[F(15,60)=2.3, p<.01]$, but no effect of sample type $[F(1,4)=1.2]$ or interaction $[F(15,60)=1.2]$. Inspection of the data for individual subjects indicated that 3 subjects showed consistently higher peck rates to element samples than to compound samples, while two did not.

\section{EXPERIMENT 2}

In Experiment 2, the delay between termination of the sample stimulus and presentation of the test stimuli was manipulated within sessions in the context of symbolic
MTS. In each of two experimental phases, four delay values were manipulated within each session. The delays varied between 0 and $4 \mathrm{sec}$. If information overload occurs during information storage, then element and compound matching accuracy should diverge with increasing delays.

\section{Method}

Subjects and Apparatus. The subjects and apparatus were the same as those used in Experiment 1.

Procedure. Sessions were identical to those of the previous experiment, with the following exceptions. The sample was presented until two pecks to the sample occurred. Following the second sample peck, the test stimuli were either presented immediately (zero-delay trials) or following a variable delay period. During nonzero delays, all three keys were dark.

There were two phases, differing in the values of the delays used. During the first phase, the delay was $0,1,2$, or $4 \mathrm{sec}$. The value of the delay was varied within sessions, using a counterbalancing procedure identical to the one used for sample duration in Experiment 1. Phase 1 was conducted for $\mathbf{4 0}$ sessions. During the second phase, the delay values were $0, .5,1$, and $2 \mathrm{sec}$. Phase 2 was conducted for 20 sessions.

\section{Results}

Mean matching accuracy for element and compound trials are shown in Figure 3, as a function of delay length during each of the two phases of the experiment. An ANOVA (delay length $x$ sample type) was carried out for each experimental phase, using the mean choice accuracy of each subject at each level of sample type and delay length. During Phase 1 , there were significant effects of delay length $[F(3,12)=9 i .2, p<.001]$ and sample type $[F(1,4)=11.4, p<.05]$, but there was no interaction between these factors $[F(3,12)<1]$. Similarly, during Phase 2, there were effects of delay length $[F(3,12)=32.6, p<.001]$ and sample type $[F(1,4)=$ $10.9, p<.05]$, but there was no interaction $[F(3,12)<1]$.

\section{DISCUSSION}

A number of previous experiments have failed to produce evidence that element and compound matching accuracy converge with increas-

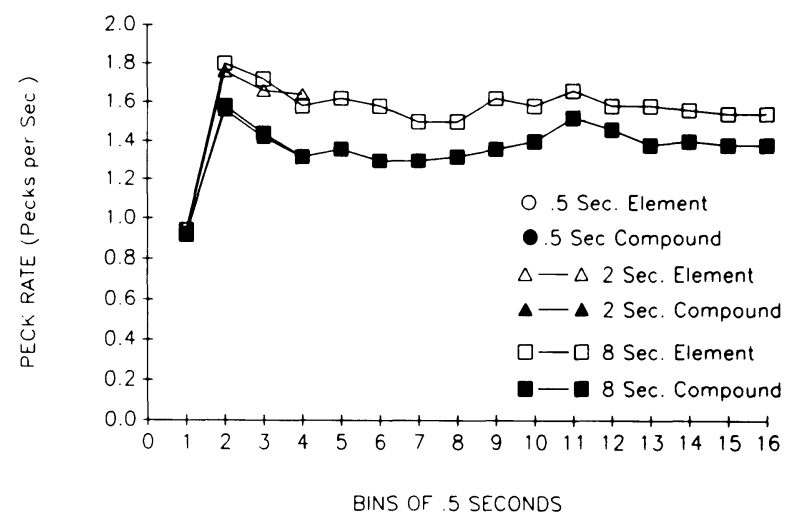

Figure 2. Mean peck rate to the sample over the course of the sample presentation period during Phase 3 of Experiment 1. The sample presentation period is divided into bins of $.5 \mathrm{sec}$ each. Data are shown separately for the 3 longest sample durations and for element and compound trials. 


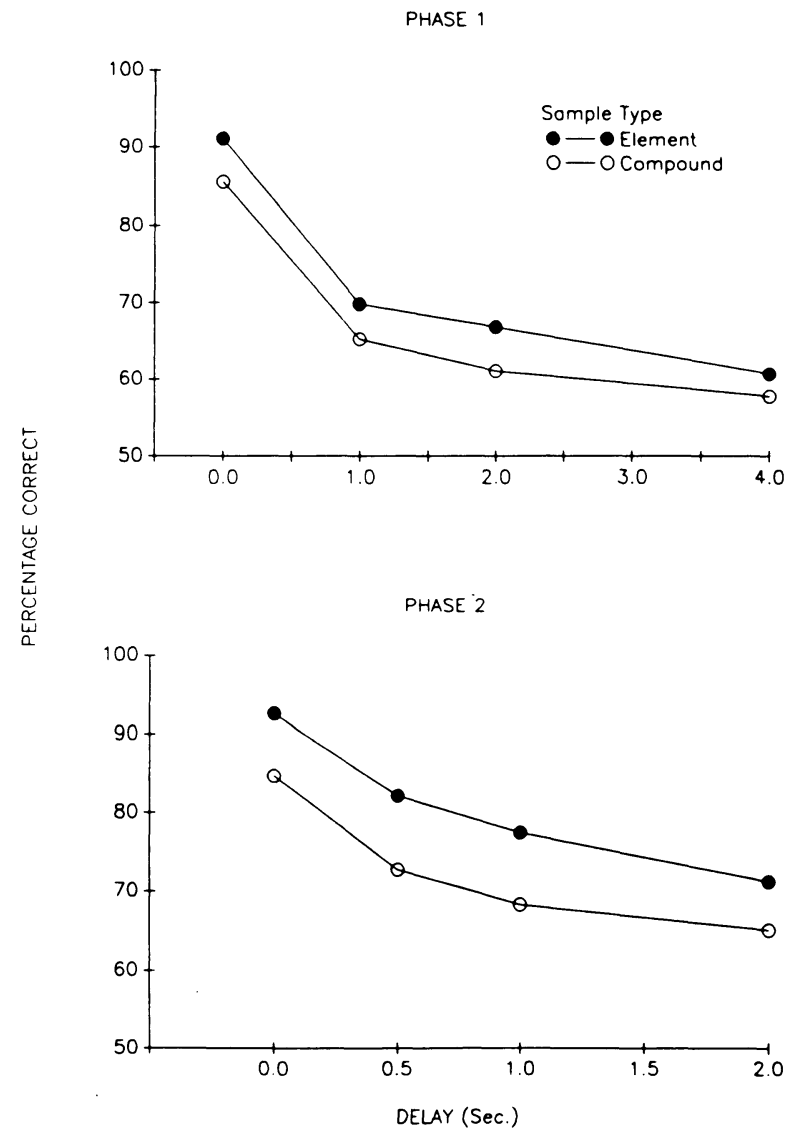

Figure 3. Mean matching accuracy as a function of delay length for element and compound trials during Phases 1 (top panel) and 2 (bottom panel) of Experiment 2.

ing sample duration or diverge with increasing delays (Brown \& Morrison, 1990; Grant \& MacDonald, 1986; Lamb \& Riley, 1981; Riley, 1984; Santi et al., 1982). It has been argued that these results provide evidence against the shared attention and memory load versions of the information overload explanation of the element superiority effect. The present results strengthen or extend this previous work in three ways. First, the element superiority effect found in the present experiments cannot be explained in terms of generalization decrement or training history. Second, in the present experiment, temporal parameters were manipulated within, rather than between, sessions, thereby rendering the sample duration on any particular trial unpredictable. Third, with respect to the first of the present experiments, it is known that high steady rates of responding to the sample occurred even throughout long sample presentation periods.

Taken together with the recent data of Brown and Morrison (1990), the present results place severe constraints on the interpretation of the element superiority effect. Although previous data have indicated a role for both generalization decrement (Cox \& D'Amato, 1982; Roberts \& Grant, 1978) and training history (Grant \& MacDonald, 1986), Brown and Morrison's (1990) data rule out the use of these as sufficient explanations for the element superiority effect found in the subjects in the present experiments. If information overload occurs at the time of sample encoding, this would seem to predict rather strongly an interaction between sample type and sample duration. The fact that, even under these improved experimental conditions, the effects of sample type and sample duration were found to be additive causes problems for the shared attention version of the information overload hypothesis. Likewise, if information overload occurs during information storage, an interaction between sample type and delay would be expected. Thus, the additivity of sample type and delay length argues against the possibility that the element superiority effect is a function of information overload during storage.

From an information processing perspective, one remaining potential locus of information overload is retrieval. During inspection of the test stimuli, the encoded and stored information must allow the choice of the correct test stimulus. It is possible that this choice process is less efficient following compound samples, owing, for example, to interference between memories for the two correct test stimuli that are mapped onto each compound sample (only one of which is present on any particular trial). Encouragement for this general view comes from the recent work of Lamb (1991). Using human subjects and reaction time as the dependent variable, Lamb found an element superiority effect analogous to that found with pigeons in MTS. Lamb's results indicate that this effect did not result from information overload at the time of information encoding, but rather from overload at a later point in information processing, such as short-term memory search. It is possible that the same is true for pigeons.

\section{REFERENCES}

Brown, M. F., Morrison, S. K. (1990). Element and compound matching-to-sample performance in pigeons: The roles of information load and training history. Joumal of Experimental Psychology: Animal Behavior Processes, 16, 185-192.

Cox, J. K., \& D'Amato, M. R. (1982). Matching to compound samples by monkeys (Cebus apella): Shared attention or generalization decrement? Journal of Experimental Psychology: Animal Behavior Processes, 8, 209-225.

Grant, D. S. (1981). Short-term memory in the pigeon. In N. E. Spear \& R. R. Miller (Eds.), Information processing in animals: Memory mechanisms (pp. 227-256). Hillsdale, NJ: Erlbaum.

Grant, D. S., MacDonald, S. E. (1986). Matching to element and compound samples in pigeons: The role of sampling coding. Journal of Experimental Psychology: Animal Behavior Processes, 12, 160-171.

LAMB, M. R. (1991). Attention in humans and animals: Is there a capacity limitation at the time of encoding? Joumal of Experimental Psychology: Animal Behavior Processes, 17, 45-54.

LAMB, M. R., \& RileY, D. A. (1981). Effects of element arrangement on the processing of compound stimuli in pigeons (Columba livia). Journal of Experimental Psychology: Animal Behavior Processes, 7, 45-58.

MAKI, W. S., \& LEITH, C. R. (1973). Shared attention in pigeons. Journal of the Experimental Analysis of Behavior, 19, 345-349.

MAKI, W. S., JR., RILEY, D. A., LeITh, C. R. (1976). The role of test stimuli in matching to compound samples by pigeons. Animal Learning \& Behavior, 4, 13-21.

RiLEY, D. A. (1984). Do pigeons decompose stimulus compounds? In H. L. Roitblat, T. G. Bever, \& H. S. Terrace (Eds.), Animal cognition (pp. 333-350). Hillsdale, NJ: Erlbaum.

RiLeY, D. A., \& LiTh, C. R. (1976). Multidimensional psychophysics and selective attention in animals. Psychological Bulletin, 83, 138-160.

RoberTS, W. A., Grant, D. S. (1978). Interaction of sample and comparison stimuli in delayed matching-to-sample with the pigeon. Journal of Experimental Psychology: Animal Behavior Processes, 4, 68-82.

Santi, A., Grossi, V., \& Gibson, M. (1982). Differences in matchingto-sample performance with element and compound sample stimuli in pigeons. Learning \& Motivation, 13, 240-256.

(Manuscript received February 14, 1991.) 